\title{
Shell like Supernova Remnants observed with Fermi-LAT
}

\author{
Francesco de Palma* \\ Instituto Nazionale di Fisica Nucleare Sezione di Torino, Turin, Italy \\ E-mail: francesco.depalmaeto.infn.it
}

\author{
Leonardo Di Venere \\ Instituto Nazionale di Fisica Nucleare Sezione di Bari, Bari, Italy, \\ E-mail: leonardo.divenereaba.infn.it
}

\section{Colin Clark}

Jodrell Bank Centre for Astrophysics, School of Physics and Astronomy, University of Manchester, Manchester M13 9PL, UK

E-mail: colin.clark-2@manchester.ac.uk

\section{on behalf of the Fermi-LAT collaboration ${ }^{\dagger}$}

\begin{abstract}
Supernova Remnants (SNRs) emitting gamma rays in the GeV-TeV energy range are key for identifying the accelerators of Galactic cosmic rays. Recently H.E.S.S. has revealed at TeV energies three new candidate shell Supernova Remnants (SNR): HESS J1534-571, HESS J1614-518 and HESS J1912+101. A radio SNR candidate has been identified as a counterpart to HESS J1534-571, therefore it is classified as a SNR. We will report on the Fermi-Large Area Telescope (LAT) analysis of HESS J1534-571 and HESS J1614-518 using 10.5 years of Pass 8 data. Within the sky region covered by HESS J1614-518, lies an additional point source, which we have identified as a new gamma-ray pulsar through the detection of pulsations in the FermiLAT data. If this new pulsar is associated with the extended emission observed by the Fermi-LAT and H.E.S.S., this hint to a classification of HESS J1614-518 as a composite SNR/PWN. An indepth analysis of the Fermi-LAT spectra of these two sources in conjunction with H.E.S.S. results can help to discriminate between hadronic and leptonic emission mechanisms. These findings could be further strengthened by future multi-wavelength observations.
\end{abstract}

36th International Cosmic Ray Conference -ICRC2019-

July 24th - August 1st, 2019

Madison, WI, U.S.A.

\footnotetext{
* Speaker.

${ }^{\dagger}$ for collaboration list see PoS(ICRC2019)1177
} 


\section{Introduction}

Supernova Remnants (SNR) are mostly detected and classified via radio and optical surveys. Some others are detected in X-ray surveys if they have a low radio emission or they lie in complex zones. Instead, the sources analyzed in this paper were identified as candidate SNR using $\mathrm{TeV}$ data of the High Energy Stereoscopic System (H.E.S.S.) telescopes and they were unknown at other wavelength [1]. Pulsar Wind Nebulae (PWN) are the largest population of identified extended Galactic sources at TeV energy while SNRs are the second ${ }^{1}$. The only method to identify a candidate SNR from other sources is to analyze its morphology. If a source has a clear shell-shape it is a strong hint that it might be an SNR. HESS J1534-571 detection in TeV was announced at 2015 ICRC conference [2], together with a reanalysis of HESS J1912+101. Both are considered candidate Supernova Remnants (SNR) for their shell-shape. In the final analysis [1] the source HESS J1614-518 was also added as a candidate SNR. HESS J1534-571 is also identified with the radio SNR G323.7-1.0.

HESS J1614-518 was the only source to be detected in 3FGL catalog, which used only 4 years of data, and it was classified as extended The other two were not detected. In the latest Fermi-LAT catalog, named 4FGL [3], that uses 8 years of data, all three sources are detected as extended. The Fermi-LAT and Magic analysis of HESS J1912+101 is reported in details in [4]. A Fermi-LAT analysis of HESS J1534-571 is also described in [5].

\section{Data Selection and analysis model}

For this analysis we use 127 months (2008 August 4 to 2019 March 3rd or MET: 239557417573340000 ) of Fermi-LAT data. We excluded time intervals in which there were bright gamma-ray burst and solar flares as it was done in the the 8 Year Source List ${ }^{2}$ (FL8Y)[3]. We use the latest Pass 8 [6] SOURCE events class (evclass=128) Fermi-LAT dataset, with Instrument Response Functions (IRFs) P8R3_SOURCE_V2. We performed the analysis using the Fermi Tools ${ }^{3}$ version 11-07-00 and fermipy ${ }^{4}$ version 0.17.4 [7].

In the following the Test Statistic is defined as $T S=2 \ln \left(L_{\max , 1} / L_{\max , 0}\right)$, where $L_{\max , 0}$ is the maximum likelihood value for a model without the additional source (the 'null hypothesis') and $L_{\max , 1}$ is the maximum likelihood value for a model with the additional source. With the same principle we define $T S_{\text {ext }}$ where the source in the hypothesis 1 is extended while in hypothesis 0 is pointlike; and $T S_{\text {curv }}$ where the source spectrum is modeled as a LogParabola (LP) in hypothesis 1 and as a PowerLaw (PL) in the hypothesis 0 .

We analyze each SNR in two energy ranges, between $1 \mathrm{GeV}$ and $2 \mathrm{TeV}$ we perform the spectral and morphological analysis of each source, while between $0.1 \mathrm{GeV}$ and $2 \mathrm{TeV}$ we do only a spectral analysis using the morphological results obtained at higher energy. This is done to exploit the better Fermi-LATangular resolution at energies above $1 \mathrm{GeV}$.

\footnotetext{
${ }^{1}$ http://tevcat.uchicago.edu/

${ }^{2}$ https://fermi.gsfc.nasa.gov/ssc/data/access/lat/fl8y/

${ }^{3} \mathrm{https}: / /$ fermi.gsfc.nasa.gov/ssc/data/analysis/software/

${ }^{4}$ https://fermipy.readthedocs.io/en/latest/index.html
} 
For the analysis above $1 \mathrm{GeV}$ we use all the events that have a zenith angle smaller than $105^{\circ}$, in order to reduce the contribution of the Earth limb. The dataset was split in the four Point Spread Function (PSF) quantiles available for the SOURCE event class ${ }^{5}$. Each PSF quantile has its own binned likelihood instance that is combined in a global likelihood function, which is then minimized. In this way each photon is analyzed with a set of IRFs that better describes its characteristics without loss in the number of photons. The region of interest (RoI) of each analysis is of $10^{\circ}$ and we use 8 energetic bins per decade and a $0.1^{\circ}$ spatial binning.

For each SNR analysis the background model used is composed of all the sources in the 4FGL, version gll_psc_v18.fit, within $15^{\circ}$ from the center of its locations reported in [1]. Given the difference in exposure time ( 8 years versus 10.5) we use the fermipy capability for searching and fitting new point sources in the RoI.We add iteratively new point sources that have a TS higher than 16 (approximately a $4 \sigma$ detection) and that are further than $0.5^{\circ}$ from another source in the model. The spectra of those models is also iteratively fitted. In order to avoid adding too many sources the iterative process ends after adding the nine most significant sources. We delete the sources that have a number of observed counts smaller than 1 , this might indeed happen since in the 4FGL the energy threshold is $50 \mathrm{MeV}$ while here we are starting from 1 $\mathrm{GeV}$. The interstellar emission model is the latest produced by the Fermi-LAT for the 4FGL, gll_iem_v07.fits. For each PSF class we use the corresponding isotropic emission model iso_P8R3_SOURCE_PSF [0/1/2/3]_V2_V1.txt, extrapolated up to $2 \mathrm{TeV}$. The point-like and extended sources in $5^{\circ}$ from the RoI center have the normalization free to vary. The spectrum of the Galactic model is described with a PL, with normalization and index kept free in the fit.

For the analysis starting at $100 \mathrm{MeV}$, we enlarged the RoI to $14^{\circ}$ and we considered 3 new binned likelihood instances leaving the other 4 unchanged except for the RoI size. This new three binned likelihood instances have an energy range between 0.1 and $1 \mathrm{GeV}$ and PSF class $1 / 2 / 3$. We drop the event class 0 because it has the worst angular resolution. We used different $z_{\max }$ selection for each PSF class: PSF1 has $z_{\max }=85^{\circ}$, PSF2 has $z_{\max }=95^{\circ}$ and PSF3 has $z_{\max }=100^{\circ}$. For all the analysis the energy dispersion was taken into account by enabling the corresponding flag in fermipy.

For the analysis described in section 4 and 5 we selected the on-phase and off-phase regions of the emission and we duplicated each binned likelihood instance. For the on-phase analysis we set the exposure of the pulsar to 1 while for the off-phase analysis we set the exposure of the pulsar to 0 . In this way it is possible to analyze correctly both the pulsar and the candidate SNR without loosing statistics. In total in the phased analysis of the HESS J1614-518 we had 8 likelihood instance above $1 \mathrm{GeV}$ and 14 above $100 \mathrm{MeV}$.

For each source we test the spatial model in the $4 \mathrm{FGL}$, a map obtained from the H.E.S.S. data, a Radial Disk and a Radial Gaussian. For the latter two we fit its position and extension using fermipy. For this part of the analysis the source has a PL spectral model. With the best spatial model we test also a LP, a BrokenPowerLaw (BPL) and a SmoothBrokenPowerLaw (SBPL) model 6 .

The systematic errors on the Spectral Energy Distribution (SED) are obtained as described in

\footnotetext{
${ }^{5}$ https://fermi.gsfc.nasa.gov/ssc/data/analysis/documentation/Cicerone/Cicerone_Data/LAT_DP.html

${ }^{6} \mathrm{https}: / /$ fermi.gsfc.nasa.gov/ssc/data/analysis/scitools/source_models.html
} 


\begin{tabular}{lccccc}
\hline \hline Name & shape & $\begin{array}{c}\text { R.A. } \\
\text { deg. }\end{array}$ & $\begin{array}{c}\text { Dec. } \\
\text { deg. }\end{array}$ & $\begin{array}{c}\text { 68\% containment radius } \\
\text { deg. }\end{array}$ & TS ext \\
\hline \hline J1534-471 & RadialDisk & $233.69 \pm 0.05$ & $-57.19 \pm 0.05$ & $0.35 \pm 0.03$ & 57.5 \\
J1534-471 & RadialGauss & $233.73 \pm 0.06$ & $-57.28 \pm 0.05$ & $0.33 \pm 0.03$ & 50.7 \\
4FGL J1533.9-5712e & RadialDisk & 233.5 & 57.2 & 0.328 & \\
\hline
\end{tabular}

Table 1: Morphological Analysis of HESS J1534-571 between $1 \mathrm{GeV}$ and $2 \mathrm{TeV}$. The last row has no errors since we use the same values that are found in the $4 \mathrm{FGL}$, we don't refit neither its size nor its location. In the 4FGL the location and the size are obtained from archival data.

detail in the appendix B of [8]. We use the eight alternative Interstellar Emission models that were developed for the Fermi-LAT SNR catalog rescaled for the use with Pass 8 data.

\section{HESS J1534-571}

The results of the analysis above $1 \mathrm{GeV}$ are listed in Table 1 and 2. From the latter table, it is evident that the best source model is the H.E.S.S. map. Both the flux and the spectral index does not seem to change much with different spatial models. The source was also clearly significantly detected with a TS of above 50. From Table 1 it is evident also that the source is clearly significantly extended. With the best spatial model we tested curved spectral models, the likelihood did not change either above $0.1 \mathrm{GeV}$ or above $1 \mathrm{GeV}$. In the $4 \mathrm{FGL}$ it is also not significantly curved and it has a hard index.

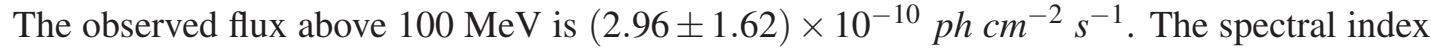
is $1.18 \pm 0.12$. Given the H.E.S.S. spectral index of $2.51 \pm 0.09_{\text {stat }} \pm 0.20_{\text {syst }}$ reported in [1] it is evident that there is a break between the energy ranges observed by the two experiment. The test statistics maps of the region of this source are in Fig. 1.

We modeled the Fermi-LAT and H.E.S.S.emission with an Inverse Compton (IC) on CMB seed photons and a hadronic model, using the Naima ${ }^{7}$ [9] simulation code. Only statistical errors were taken into account. In order to estimate the acceleration efficiencies in the two cases, we set the source distance to $3.5 \mathrm{kpc}$, following[10], and the ambient density to $1 \mathrm{~cm}^{-3}$. The results are in Table 5. The hadronic and leptonic model have a similar Bayesian information criterion (BIC).

\section{PSR J1615-5137}

The first version of this analysis used as background source list the 3FGL. In that catalog the HESS J1614-518 region was modeled only as a RadialDisk. During the various analyses with a single source with various extended shapes we found out that there was always a strong point like residual that could be well modeled with a curved spectrum. We performed a blind search for pulsations from the new point source, and detected a highly significant signal, identifying it as a new gamma-ray pulsar. There is not a counterpart of this PSR at other wavelength. This point source was later found inside the extended emission in the Preliminary LAT 8-year Point Source

\footnotetext{
${ }^{7} \mathrm{https}: / /$ naima.readthedocs.io/en/latest/
} 


\begin{tabular}{|c|c|c|c|c|}
\hline Name & shape & $\begin{array}{c}\text { flux } \\
p h \mathrm{~cm}^{-2} \mathrm{~s}^{-1}\end{array}$ & index & TS \\
\hline J1534-471 & map & $2.16 \mathrm{e}-10 \pm 1.16 \mathrm{e}-10$ & $-1.251 \pm 0.190$ & 64.1 \\
\hline J1534-471 & adialDisk & $2.14 \mathrm{e}-10 \pm 7.84 \mathrm{e}-11$ & $-1.253 \pm 0.119$ & 61.8 \\
\hline J1534-471 & RadialGauss & $2.03 \mathrm{e}-10 \pm 7.34 \mathrm{e}-11$ & $-1.251 \pm 0.116$ & 57.2 \\
\hline 4FGL J1533.9-5712e & RadialDisk & $2.02 \mathrm{e}-10 \pm 8.42 \mathrm{e}-11$ & $-1.255 \pm 0.148$ & 54.0 \\
\hline
\end{tabular}

Table 2: Spectral Analysis of HESS J1534-571 between $1 \mathrm{GeV}$ and $2 \mathrm{TeV}$. The preferred model for this source is the H.E.S.S. map, since it gives the highest likelihood with the lowest number of spatial degrees of freedom.
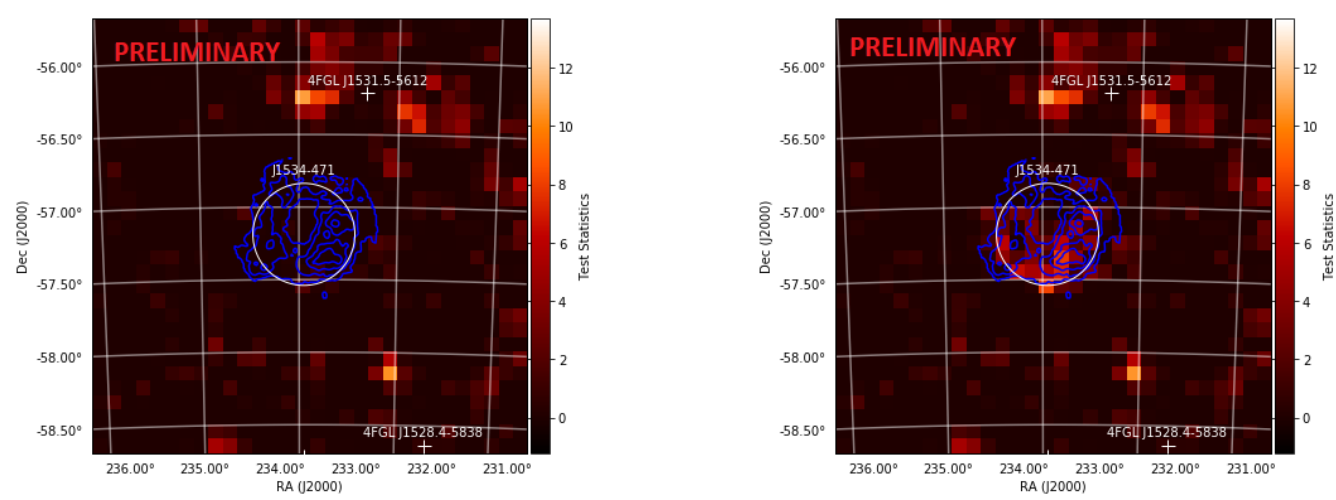

Figure 1: TS maps of HESS J1534-571. In blue the H.E.S.S. contour map, in white the best Radial Disk model optimized in the fit. The rest of white sources are background sources in the 4FGL. On the left the SNR is in the model, on the right it is excluded.

List $^{8}$ and in the final version of the 4FGL. It should be noted that in the 2FGL[11] the emission of the region was modeled with only two point sources, one was placed really near the location we found for the PSR. In all the catalogs it had a curved spectra but the identification of it as a gamma-ray pulsar was done in this work.

The weighted counts of the PSR J1615-5137 are shown in Fig. 2. We use a PowerLawSuperExpCutoff as a spectral model of the PSR as it is used in the 4FGL. The total flux above 100 $\mathrm{MeV}$ of the PSR is $2.01 \pm 0.18 \times 10^{-08} \mathrm{ph} \mathrm{cm}^{-2} \mathrm{~s}^{-1}$, its location is R.A. $-51.62^{\circ}$ Dec. $243.82^{\circ}$ obtained from the pulsation analysis. We did not find any significant proper motion from the timing analysis. Assuming that a full conversion between spin-down power $\left(\mathrm{Edot}=7.3 \times 10^{34} \mathrm{erg} / \mathrm{s}\right)$ and gamma-ray flux, we can estimate an upper limit distance of $4.1 \mathrm{kpc}$.

\section{HESS J1614-518}

The results of the analysis above $1 \mathrm{GeV}$ of the HESS J1614-518 done with the phased analysis described in Section 2 are listed in Table 3 and 4. From the latter table, it is evident that the best source model is the Radial Gaussian. The spectral index does not seem to change much with

\footnotetext{
${ }^{8}$ https://fermi.gsfc.nasa.gov/ssc/data/access/lat/fl8y/
} 


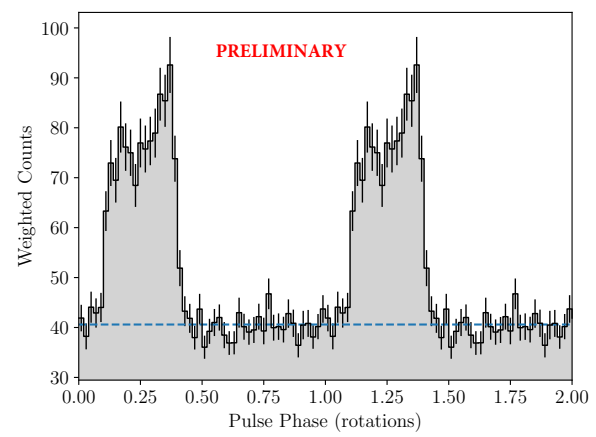

Figure 2: Gamma-ray pulse profile of the newly-detected PSR J1615-5137. For the phased analysis described in Section 4, we defined the on- and off-phase selections as phases 0 to 0.5 , and 0.5 to 1 , respectively.

\begin{tabular}{lccccc|}
\hline \hline name & shape & $\begin{array}{c}\text { R.A. } \\
\text { deg. }\end{array}$ & $\begin{array}{c}\text { Dec. } \\
\text { deg. }\end{array}$ & $\begin{array}{c}\text { 68\% containment radius } \\
\text { deg. }\end{array}$ \\
\hline \hline J1614-518 & RadialDisk & $243.64 \pm 0.02$ & $-51.83 \pm 0.02$ & $0.38 \pm 0.01$ & 378.6 \\
J1614-518 & RadialGauss & $243.72 \pm 0.03$ & $-51.77 \pm 0.03$ & $0.56 \pm 0.03$ & 454.9 \\
4FGL J1615.3-5146e & RadialDisk & 243.83 & -51.78 & 0.42 & \\
\hline
\end{tabular}

Table 3: Morphological Analysis of HESS J1614-518 between $1 \mathrm{GeV}$ and $2 \mathrm{TeV}$. The last row has no errors since we use the same values that are found in the $4 \mathrm{FGL}$, we don't refit neither its size nor its location. In the 4FGL the location and the size are obtained from archival data.

different spatial models, while the flux with the Radial Gaussian model is larger. The source was also clearly significantly detected with a TS of above 400. From Table 3 it is evident also that the source is clearly significantly extended. With the best spatial model we tested curved spectral models. In the analysis above $1 \mathrm{GeV}$ no curvature is observed, while in the analysis starting from $100 \mathrm{MeV}$ we find $T S_{\text {curv }}=33$ with the LP model. Similar results but with an higher number of degrees of freedom was obtained with the BPL and the SBPL. Using the alternative IEMs this significance drops at around 3 sigma for almost all the 8 models. In the 4FGL it is also significantly curved and modeled with a LP. The flux above $100 \mathrm{MeV}$ is $1.39 \pm 0.17 \mathrm{ph} \mathrm{cm}^{-2} \mathrm{~s}^{-1}$ with spectral parameters: $\alpha=1.23 \pm 0.4$ and $\beta=0.090 \pm 0.006$. This LP seems to be smoothly connected to the H.E.S.S. data. The test statistic maps of this analysis are shown in Fig. 3.

We modeled the Fermi-LAT and H.E.S.S.emission with an Inverse Compton (IC) on CMB seed photons and a hadronic model, using the Naima [9] simulation code. Only statistical errors were taken into account. In order to estimate the acceleration efficiencies in the two cases, we set the source distance to $1 \mathrm{kpc}$ (similar of what the authors estimate in [1] using possible associations), and the ambient density to $1 \mathrm{~cm}^{-3}$. The results are in Table 5 . The hadronic model has a better BIC.

\section{Conclusion}

In this work we report the the detailed morphological and spectral analysis of two candidate shell-like SNRs. Both sources are clearly detected and characterized. Given the presence of the 


\begin{tabular}{|c|c|c|c|c|}
\hline Name & shape & $\begin{array}{c}\text { flux } \\
p h \mathrm{~cm}^{-2} \mathrm{~s}^{-1}\end{array}$ & index & TS \\
\hline J1614-518 & map & $4.28 \mathrm{e}-09 \pm 2.56 \mathrm{e}-10$ & $-1.757 \pm 0.023$ & 481.9 \\
\hline J1614-518 & RadialDisk & $4.75 \mathrm{e}-09 \pm 5.69 \mathrm{e}-10$ & $-1.772 \pm 0.052$ & 497.6 \\
\hline J1614-518 & RadialGisk & $8 \mathrm{e}-09 \pm 4.97 \mathrm{e}-10$ & $-1.816 \pm 0.024$ & 620.4 \\
\hline 4FGL J1615.3-5146e & RadialDisk & $4.43 \mathrm{e}-09 \pm 2.68 \mathrm{e}-10$ & $-1.786 \pm 0.021$ & 470.1 \\
\hline
\end{tabular}

Table 4: Spectral Analysis of HESS J1614-518 between $1 \mathrm{GeV}$ and $2 \mathrm{TeV}$. The preferred model for this source is the Radial Gaussian, since it gives the highest likelihood with the lowest number of spatial degrees of freedom.
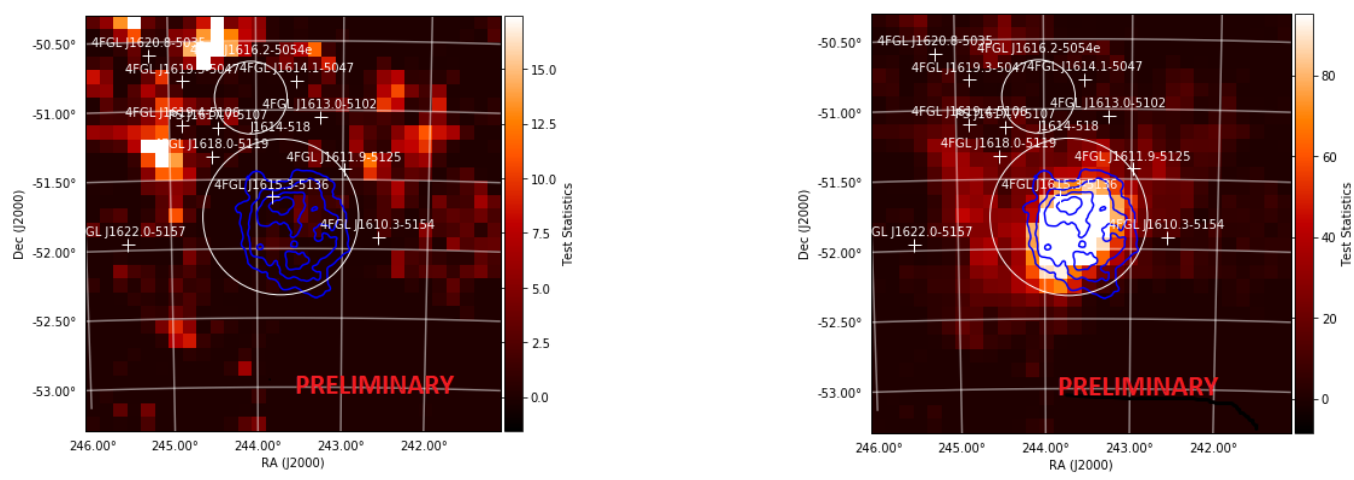

Figure 3: TS maps of HESS J1614-518. In blue the H.E.S.S. contour map, in white the Radial Gaussian model optimized in the fit. The rest of white sources are background sources in the 4FGL. In this plot the new PSR J1615-5137 is shown with its 4FGL name of 4FGL J1615.3-5136. On the left the SNR is in the model, on the right it is excluded.

\begin{tabular}{lccccc}
\hline \hline source name & model & index & $\begin{array}{c}\text { cutoff energy } \\
{[\mathrm{TeV}]}\end{array}$ & $\begin{array}{c}W_{p} \text { or } W_{e} \\
\text { erg }\end{array}$ & BIC \\
\hline \hline HESS J1534-571 & IC & $1.43_{-0.34}^{+0.11}$ & $9.92_{-2.29}^{+3.02}$ & $\left(2.9_{-0.8}^{+2}\right) \times 10^{47}$ & 21.6 \\
HESS J1534-571 & hadronic & $1.18_{-0.18}^{+0.10}$ & $14.70_{-4.66}^{+6.31}$ & $(9.9 \pm 0.9) \times 10^{49}$ & 21.1 \\
\hline HESS J1614-518 & IC & $2.51_{-0.07}^{+0.06}$ & $16.48_{-2.02}^{+3.02}$ & $\left(8_{-2}^{+4}\right) \times 10^{48}$ & 83.20 \\
HESS J1614-518 & hadronic & $1.91_{-0.03}^{+0.02}$ & $31.46_{-6.24}^{+8.70}$ & $\left(6.91_{-0.3}^{+0.2}\right) \times 10^{49}$ & 77.03 \\
\hline
\end{tabular}

Table 5: Fit obtained using Naima [9] on the joined Fermi-LAT and H.E.S.S. SED. $W_{p}$ and $W_{e}$ are evaluated for particles with energies above $1 \mathrm{GeV}$.

new PSR J1615-5137 inside the emission of HESS J1614-518 it is possible that the emission is actually not related to the SNR but to a PWN, further studies will be needed.

\section{Acknowledgements}

The Fermi-LAT Collaboration acknowledges support for LAT development, operation and data analysis from NASA and DOE (United States), CEA/Irfu and IN2P3/CNRS (France), ASI 
and INFN (Italy), MEXT, KEK, and JAXA (Japan), and the K.A. Wallenberg Foundation, the Swedish Research Council and the National Space Board (Sweden). Science analysis support in the operations phase from INAF (Italy) and CNES (France) is also gratefully acknowledged. This work performed in part under DOE Contract DE-AC02-76SF00515. C.J.C. acknowledges support from the ERC under the European Union's Horizon 2020 research and innovation programme (grant agreement No. 715051; Spiders).

\section{References}

[1] H. E. S. S. Collaboration, H. Abdalla, A. Abramowski, F. Aharonian, F. Ait Benkhali, A. G. Akhperjanian et al., A search for new supernova remnant shells in the Galactic plane with H.E.S.S., Astronomy \& Astrophysics 612 (2018) A8 [1801.06020].

[2] G. Puehlhofer, F. Brun, M. Capasso, R. Chaves, C. Deil, A. Djannati-Ataï et al., Search for new supernova remnant shells in the Galactic plane with H.E.S.S., in 34th International Cosmic Ray Conference (ICRC2015), vol. 34, p. 886, Jul, 2015, 1509.03872.

[3] The Fermi-LAT collaboration, Fermi Large Area Telescope Fourth Source Catalog, arXiv e-prints (2019) arXiv:1902.10045 [1902.10045].

[4] T. Nagayoshi, D. Green, F. de Palma and Fermi-LAT Collaboration, Gamma-ray emission from HESS J1912+101 observed by MAGIC and Fermi-LAT, International Cosmic Ray Conference (This conference) (2019) .

[5] M. Araya, Detection of GeV Gamma-Rays from HESS J1534-571 and Multiwavelength Implications for the Origin of the Nonthermal Emission, The Astrophysical Journal 843 (2017) 12 [1705.03902].

[6] P. Bruel, T. H. Burnett, S. W. Digel, G. Johannesson, N. Omodei and M. Wood, Fermi-LAT improved Pass 8 event selection, arXiv e-prints (2018) arXiv:1810.11394 [1810.11394].

[7] M. Wood, R. Caputo, E. Charles, M. Di Mauro, J. Magill, J. S. Perkins et al., Fermipy: An open-source Python package for analysis of Fermi-LAT Data, International Cosmic Ray Conference 35 (2017) 824 [1707.09551].

[8] F. Acero, M. Ackermann, M. Ajello, L. Baldini, J. Ballet, G. Barbiellini et al., The First Fermi-LAT Supernova Remnant Catalog, The Astrophysical Journal Supplement Series 224 (2016) 8.

[9] V. Zabalza, Naima: a Python package for inference of relativistic particle energy distributions from observed nonthermal spectra, Proc. of International Cosmic Ray Conference 2015 (2015) 922 [1509.03319].

[10] N. I. Maxted, C. Braiding, G. F. Wong, G. P. Rowell, M. G. Burton, M. D. Filipović et al., Searching for an interstellar medium association for HESS J1534 - 571, Monthly Notices of the Royal Astronomical Society 480 (2018) 134 [1807.01300].

[11] P. L. Nolan, A. A. Abdo, M. Ackermann, M. Ajello, A. Allafort, E. Antolini et al., Fermi Large Area Telescope second source catalog, The Astrophysical Journal Supplement Series 199 (2012) 31. 